\title{
hsTropI: an early biomarker of acute coronary syndrome \& MI
}

\author{
Neogi S.S. ${ }^{1}$, Kapoor R.K. ${ }^{2}$ \\ ${ }^{1}$ Dr Sohini Sengupta Neogi, ${ }^{2}$ Dr Raj Kumar Kapoor; both authors are affiliated with Department of Biochemistry, BLK \\ Superspeciality Hospital, Pusa Road, Rajinder, Nagar, New Delhi, India.
}

Address for Correspondence: Dr Sohini Sengupta Neogi, E-mail: drsohini9@ gmail.com

\begin{abstract}
In the Emergency Department cardiac troponins are the preferred biomarkers for the diagnosis of acute myocardial infarction (MI) and are useful for risk stratification of patients with acute coronary syndrome (ACS) and stable ischemic heart disease. The introduction of 'high sensitivity' Troponins (hscTn) in many tertiary care centers has transformed the diagnostic scenario, wherein, the time from onset of chest pain to its diagnosis has significantly been reduced. With the advent of new hsTnI assays, which have a low limit of detection, low imprecision and low reference limits, the possibility of more patients with unstable angina being classified as having non-ST-elevation myocardial infarction (NSTEMI) will now increase. Its use will identify more high-risk patients with undifferentiated chest pain, that will help 'rule-in' or 'rule-out' acute myocardial infarction. In conclusion, it may be said that hsTropI is a sensitive, albeit less specific marker of MI. In patients of mildly elevated hsTropI and without evidence of ST elevation, a serial assessment of the biomarker is suggested. This will likely translate into more improved outcomes in this difficult patient population when ECG findings and clinical presentation do not suggest a clear diagnosis.
\end{abstract}

Keywords: hs TroponinI (hsTropI), acute coronary syndrome, Non ST elevation MI

\section{Introduction}

Cardiovascular disease is the leading cause of death worldwide and coronary artery disease is its most prevalent manifestation. In clinical practice cardiac troponins (cTn) are the cornerstone of diagnosis, risk stratification and selection of optimal treatment strategy in patients with acute coronary syndrome. According to the third update of the universal definition of myocardial infarction (MI), cTn is the preferred cardiac biomarker of myocardial necrosis in the setting of acute myocardial ischemia.

Cardiac troponins I (cTnI) and T (cTnT) have for long, been used as standard biomarkers for detection of myocardial injury, for risk stratification in patients suspected of acute coronary syndrome, and for the diagnosis of myocardial infarction. However, there is increasing evidence based on recent clinical database, which favours the use of highsensitivity (hs) troponin assays as the initial marker for acute cardiac conditions $(1,2)$.

The purpose of this review is to analyse the significance of the prefix 'hs' in the background of the biochemistry of cTnI and to address the issues arising from biological variation, assay imprecision characteristics and possible 'rule-in' and 'rule-out' of myocardial infarction using hs troponin I assays.

Human cTnI occurs in cardiac muscle tissue as a single isoform of 209 amino acid residues, with a molecular weight of approximately 23-24 kDa. Three human cTnI isoforms have been described: one is produced in cardiac muscle (cTnI), and one isoform each is produced in slow-twitch and fast-twitch skeletal muscles (slow sTnI and fast sTnI, respectively) (3).

Manuscript received: $7^{\text {th }}$ January 2017

Reviewed: $14^{\text {th }}$ January 2017

Author Corrected: $20^{\text {th }}$ January 2017

Accepted for Publication: $28^{\text {th }}$ January 2017 


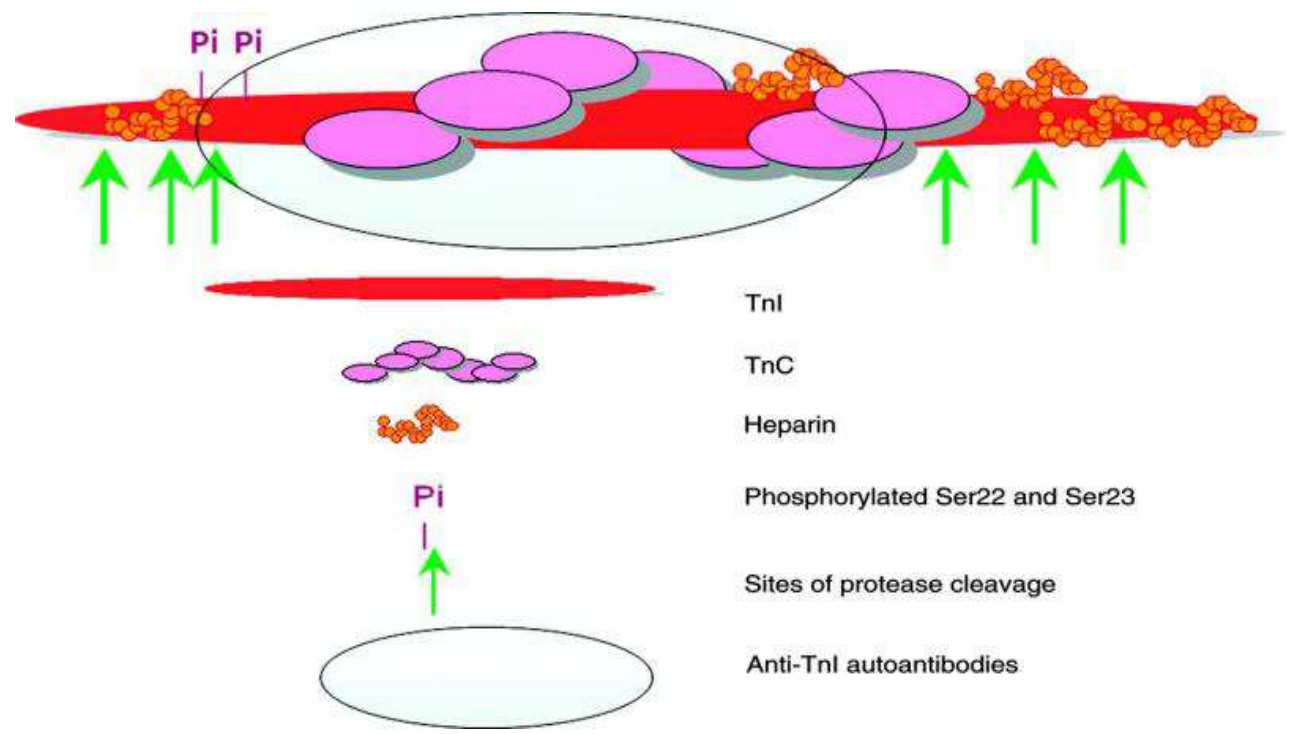

Fig-1: Arrangement of cTropI in the cardiac tissue

Significance of 'hs': The term "high sensitivity" reflects the characteristics of the assay and does not refer to a difference in the form of cardiac troponin being measured. Several names have been used in the literature for these assays, including "high-performance," "highly sensitive," "high-sensitive," "ultrasensitive," "novel highly sensitive," "sensitive," and "high sensitivity." In a scorecard concept, an assay is proposed to be 'high sensitivity' or "hs' if it meets 2 basic criteria (4). First, the total imprecision (CV) at the 99th percentile value should be $\leq 10 \%$. Second, measurable concentrations below the 99th percentile should be attainable with the assay at a concentration value above the assay's limit of detection for at least $50 \%$ (and ideally >95\%) of healthy individuals to attain the highest level of scorecard designation. Troponin assays have been developed with successive generations such that they can detect increasingly low levels of troponin (5).

This has incrementally reduced the diagnostic cut-offs for the rule-out of MI from $0.5 \mu \mathrm{g} / \mathrm{L}$ in the first generation assays to the levels of $0.05-0.1 \mu \mathrm{g} / \mathrm{L}$ (3rd generation), which are in widespread use today. However, the recent development of high-sensitivity troponin (hsTn) assays, which are in use in a few centres, can detect levels as low as $0.003 \mu \mathrm{g} / \mathrm{L}$ (3 $\mathrm{ng} / \mathrm{L}$ ). The highly sensitive troponin assays remove the need to wait for several hours after the onset of chest pain symptoms required by previous assays in order to reliably detect enough circulating troponin to confirm or exclude MI. It also means that the concept of a 'negative' troponin becomes relatively redundant as troponin at some level can almost always be detected even in healthy patients. These concepts will therefore, have widespread clinical implications. The diagnostic sensitivity of hsTn assays (ability to rule-out MI) are of the order of 90-95\% when tested at the point of admission $(6,7)$. When compared with older assays this is a marked improvement, but it still allows the potential for missing $5-10 \%$ of all MIs, with potentially adverse consequences for patient care.

The counterpoint to the excellent sensitivity of hsTn testing is a lower specificity (ability to rule-in MI). Although cardiac troponin is, by definition, completely specific for myocardial injury, it is not specific for the diagnosis of acute MI. This leads to a problem for hsTn where specificity has been reported to be $80-85 \%$ (6). Phosphorylation of cardiac troponin I (cTnI) by protein kinase C (PKC) is implicated in cardiac dysfunction. Recently, Serine 199 (Ser199) was identified as a target for PKC phosphorylation and increased Ser199 phosphorylation occurs in end-stage failing human myocardium compared to the non-failing myocardium. Ser199 pseudo-phosphorylation decreased cTnI binding to both actin and actin-tropomyosin.

Moreover, altered susceptibility of cTnI to proteolysis by calpain I was found when Ser199 was pseudo-phosphorylated. Published data demonstrate that low levels of cTnI-Ser199 pseudo-phosphorylation ( 6\%) increase myofilament $\mathrm{Ca}$ (2+)-sensitivity in human cardiomyocytes, most likely by decreasing the binding affinity of cTnI for actin-tropomyosin. In addition, cTnI-Ser199 pseudo-phosphorylation or mutation regulates calpain I mediated proteolysis of cTnI (8). 
Gender specificity of hs TropI: Gender is an important determinant of cardiovascular risk, and men generally develop cardiovascular disease earlier than women. Increased levels of hs-TnIhave been shown to be predictive of cardiovascular death, with stronger effects in women. However, it remains unclear whether the stronger association between hs-TnI and cardiovascular death in women is based on the ability of hs-TnI to predict myocardial infarction (MI) or heart failure (HF). One study aimed at assessing the influence of gender on the association between levels of hs-TnI and incidence of MI and HF. hs-TnI was measured in 5,060 women and 4,054 men (all free from known cardiac disease at baseline) participating in the prospective observational study. The C-index for hs-TnI was stronger for women than men for MI ( $\mathrm{p}<0.001$ ), and it was concluded that in the general population, the association between hs-TnI concentrations and MI is stronger in women than in men. For HF, the impact of gender on the prognostic value of hs-TnI was found to be less pronounced. Increased levels of troponin I in women may thus reflect an adverse phenotype more prone to the development of cardiovascular disease (9).

Another study showed sex-dependent differences in hsTnI in the 99th percentile of healthy population, with concentrations in women being approximately 50\% lower. Although increasing the proportion of women with increased troponin I, adopting sex-specific cutoffs with the hs-TnI assay did not lead to an increase in AMI diagnoses in females, or in the number of women undergoing angiography (10). Another study included 2077 adults from the general population aged 25-41 years without cardiovascular disease. cTnI was measured using a high-sensitivity assay. A stepwise backward linear regression analyses was performed to identify variables independently associated with hs-TnI levels. Sex, age, and systolic blood pressure were found to be the strongest determinants of hs-TnI in healthy adults. The 99th percentile was three times higher in men compared to women. Hence, it was concluded that sex-specific cut-off values may be preferable when applying hs-TnI for screening purposes (11).

'Rule in' and 'Rule out' of AMI: In a prospective, observational study of consecutive patients presenting to emergency departments where high-sensitivity cardiac troponin I (hs-TnI) was measured on clinical indication, the negative predictive value (NPV) and diagnostic sensitivity of an hs-TnI concentration < limit of detection (LoD) at presentation was determined for acute myocardial injury and for AMI or cardiac death at 30 days. It was observed that a single hs-TnI concentration $<$ LoD rules out acute myocardial injury, regardless of etiology, with an excellent NPV and diagnostic sensitivity, and identifies patients at minimal risk of AMI or cardiac death at 30 days (12). Another study compared the incidence of undetectable (below the limit of detection (LoD)), measurable (LoD to 99th percentile), and increased cTnI (concentrations above the 99th percentile) between high-sensitivity cTnI (hs-TnI) and contemporary cTnI assays. It was concluded that the hs-TnI assay provides clinicians with more numeric cTnI concentrations. This occurs via a shift from results below the LoD to those between the LoD and the 99th percentile and does not increase the number of cTnI concentrations above the 99th percentile (13). Early diagnosis of acute myocardial infarction (AMI) can ensure quick and effective treatment but only $20 \%$ of adults with emergency admissions for chest pain have an AMI. hs-cTn assays may allow rapid rule-out of AMI and avoidance of unnecessary hospital admissions and anxiety. Eighteen studies were included in a clinical effectiveness review.

The optimum strategy used a limit of blank (LoB) or limit of detection (LoD) threshold (depending on the manufacturers guidelines) in a presentation sample to rule out AMI. Patients testing positive were then subjected to a further test at 2 hours with a conclusion that a result above the 99th centile on either sample and a delta $(\Delta)$ of $\geq 20 \%$ has some potential for ruling in an AMI whereas a result below the 99th centile on both samples and a $\Delta$ of $<20 \%$ can be used to rule out an AMI. There was some evidence to suggest that hs-cTn testing may provide cost-effective approach to early rule-out of AMI. However, further research is needed to clarify optimal diagnostic thresholds and testing strategies (14). The early triage of patients toward rule-out and rule-in of acute myocardial infarction (AMI) is challenging. Therefore, one study aimed at developing a 2-h algorithm high-sensitivity cardiac troponin I (hs-TnI). hs-TnI was measured at presentation and after $2 \mathrm{~h}$ in a blinded fashion. A simple algorithm incorporating hs-TnI baseline values and absolute 2-h changes allowed a triage toward safe rule-out or accurate rule-in of AMI in the majority of patients (15). International guidelines recommend that early serial sampling of high sensitivity troponin be used to accurately identify AMI in patients presenting with chest pain. The background evidence for this approach is limited. One study evaluated whether on presentation and 4-hour hs-TnI could be used to accurately rule-out AMI. This study concluded that hs-TnI $>99$ th percentile thresholds measured on presentation and at 4-hours was not a safe strategy to rule-out AMI in this clinical 
setting irrespective of whether sex-specific 99th percentiles were used, or whether hs-TnI was combined with ECG results (16). Another multicentric study aimed to prospectively derive and validate a novel $1 \mathrm{~h}$-algorithm using hs-TnI for early rule-out and rule-in of acute myocardial infarction. Using a simple algorithm incorporating baseline hs-TnI values and the absolute change within the first hour allows safe rule-out as well as accurate rule-in of acute myocardial infarction in $70 \%$ of patients presenting with suspected acute myocardial infarction (17). Another study evaluated the incidence of major adverse cardiac events (MACE) at 1 year in emergency department (ED) patients with possible acute coronary syndromes, stratified by high hs-TnI concentrations using sex-specific cut points compared with overall cut points. It was observed that sex-specific cut points improve the identification of women but not men at risk for 1-year MACE and the net-effect across the whole ED population with possible cardiac chest pain was minimal. It was suggested that lowering the clinical cut-off point for both sexes may be appropriate for prognostic purposes (18). In a prospective multicenter diagnostic study, 1,500 patients presenting with suspected AMI to the emergency department were enrolled. The final diagnosis was centrally adjudicated by 2 independent cardiologists blinded to hs-TnI concentrations. The hsTnI 0-/1-hour algorithm incorporated measurements performed at baseline and absolute changes within 1 hour.The study concluded that the hs-TnI 0-/1-hour algorithm performs very well for early rule-out as well as rule-in of AMI and may be used as a safe and effective approach to substantially reduce the time to diagnosis (19).

Assessment of patients with suspected non-ST elevation myocardial infarction (NSTEMI) is based on cardiac troponin (cTn) levels with the 99th percentile as cut-off. However, cardiovascular risk starts already at lower troponin concentrations. The utility of 2-hour algorithms using the hs-TnI 97.5th percentile as cut-off was estimated. It concluded that the hs-TnIat 97.5th percentile integrated into 2-hour algorithms provided high diagnostic estimates and could, due to better prognostic properties serve as an alternative to the 99th percentile (20). A prospective, multicentric study was conducted to analyse whether levels of hs-cTn below their respective 99th percentile can be used as a single parameter to rule out AMI at presentation. hs-cTn was measured using four different methods (hs-cTnT Roche, hs-TnI Siemens, hsTnI Beckman Coulter and hs-TnI Abbott) in consecutive patients presenting to the emergency department with acute chest pain and the patients were followed for death or AMI during a mean period of 24 months. It was concluded that normal hs-TnI levels at presentation should not be used as a single parameter to rule out AMI as $6 \%-23 \%$ of adjudicated AMI cases had normal levels of hs-cTn levels at presentation. It also highlighted the lack of standardisation among hsTnI assays resulting in substantial differences in sensitivity and NPV at the 99th percentile (21). In another study, a thirdgeneration high sensitivity "guidelines acceptable" troponin I assay (hs-TnI) was evaluated against a contemporary "clinically usable" troponin assay (cTnI). Baseline and 90-minute samples of patients suspected to be suffering from acute coronary syndrome were analyzed for cTnI and hs-TnI. Sensitivity, specificity, positive and negative predictive values for AMI and 30-day adverse cardiac events (ACE) were compared.The hs-TnI assay achieved a 90-minute rule out for AMI and detected more than three times as many AMI cases. The specificity increased with the $\Delta 30 \%$ criteria. The hs-TnI assay also detected more cases of patient at risk for adverse cardiac events at 30 days (22). Several assays for the measurement of cardiac troponin (cTn) are available, but differences in their analytical performances may affect the diagnosis of acuteMI.A survey was conducted at all Danish departments of clinical biochemistry at hospitals receiving patients with suspected acute MI to gather information about the assay and cut-off value used. It was concluded that several assays for the measurement of cardiac troponin (cTn) are available, but differences in their analytical performances may affect the diagnosis of MI (23).

Factors influencing levels of hsTropI: In another study conducted to ascertain correlates of hs-TnI and its incremental prognostic utility for incident coronary heart disease (CHD) among older asymptomatic subjects, hs-TnI was measured in stored baseline serum samples. After a median follow-up of 11.3 years, $164 \mathrm{CHD}$ events were documented. The most significant correlates of hs-TnI were black race, body mass index, hypertension, LDL cholesterol and estimated glomerular filtration rate (eGFR) (24). In another study, blood was collected from apparently healthy individuals and the outcome as to all-cause death and incidence of cardiovascular disease (CVD) and coronary heart disease (CHD) was followed up to 10 years. It suggested that hs-TnI reflects the status of the myocardium even in seemingly healthy individuals and that the measurements of hs-TnI may be useful for primary prediction of heart disease. This could form the basis of future prospective clinical trials for determining whether measuring hs-TnI can be used in the prevention of CVD/CHD (25). Another study compared the diagnostic accuracy of conventional troponin/traditional coronary artery disease (CAD) assessment and hsTn I/advanced CAD assessment for acute coronary syndrome (ACS) during the index 
hospitalization. It was concluded that hsTnI at the time of presentation followed by early advanced coronary CTA assessment improves the risk stratification and diagnostic accuracy for ACS as compared to conventional troponin and traditional coronary CTA assessment (26). In another study, the relationship of cardiac troponin (cTn) levels with conventional and ambulatory blood pressure (BP) in young and healthy adults was evaluated. Using a hs assay, hs-TnI was detectable in virtually all participants of a young and healthy population. hs-TnI was independently associated with systolic BP and left ventricular hypertrophy (27). Another study was conducted to determine whether hs-TnI, which is detectable in a higher proportion of normal subjects than hsTnT, is associated with a major adverse cardiovascular event (MACE) in patients of type 2 diabetes mellitus. It demonstrated that elevated hs-TnI in patients with T2DM is associated with increased MACE, HF, MI and cardiovascular mortality. Importantly, a normal hs-TnI level has an excellent negative predictive value for future adverse cardiovascular events during long-term follow-up (28). Another study was designed to evaluate simultaneously conventional cTn together with their corresponding highly sensitive determinations in stable hemodialysis (HD) patients. Ability of cTn to stratify HD patient risk was assessed. A large proportion of patients free of acute coronary syndrome (ACS) were found to have hs-cTn Ihigher than the 99th percentile which could be seen as a limiting factor for ACS screening (29).

Patients with end-stage renal failure were found to exhibit a chronic elevation of serum cardiac troponin (cTn) concentration. In order to facilitate the diagnosis of myocardial infarction in these patients, it is necessary to distinguish an increased cTn concentration due to a acute event, from that being a manifestation of chronic elevation. It was seen that long-term biological variation of $\mathrm{cTn}$ in stable haemodialysis patients is similar to that in healthy individuals and in patients with stable coronary arterial disease. Serial measurements are required to detect significant changes in cTn concentrations and support diagnosis of myocardial infarction in these patients (30). A well-characterized communitybased cohort of 2042 study participants underwent clinical assessment and echocardiographic evaluation. Baseline hs-TnI measurements were obtained in 1843 individuals. Measurable hs-TnI was identified in 93\% of the community-based study cohort and $88 \%$ of the healthy reference cohort.

Parameters that significantly contributed to higher hs-TnI concentrations in the healthy reference cohort included age, male sex, systolic blood pressure, and left ventricular mass. Glomerular filtration rate and body mass index were not independently associated with hs-TnI in the healthy reference cohort. Individuals with diastolic and systolic dysfunction, hypertension, and coronary artery disease (but not impaired renal function) had significantly higher hs-TnI values than the healthy reference cohort (31).

\section{Conclusion}

It has been seen generally that chest pain is a common presenting symptom in the emergency department; however, the majority of these chest pain admissions are not due to AMI. AMI can be life threatening and early diagnosis or rule out of AMI might potentially improve morbidity and mortality, as well as reduce time to decision and overall treatment costs. Over the last few years newer hs-cTnassays have been developed that are more sensitive than conventional assays, have low limit of detection, low imprecision and low reference limits.

The development of hsTropI assays enables precise quantification of extremely low troponin concentrations. Such hs-tropI assays are recommended in early rule-out protocols for AMI, when measured at presentation and again at 3-6 h. With the advent ofnew hs-TnI assays, more patients with unstable angina will be classified as having non-ST-elevation myocardial infarction. The use of hs-TnI assays will identify more high-risk patients among millions who present to the Emergency Departments each year with undifferentiated chest pain. This will likely translate into more appropriate care and improved outcomes in this difficult patient population when ECG findings and clinical presentation do not suggest a clear diagnosis. Recent studies have shown that their utilization seems to improve the diagnostic accuracy in detecting $\mathrm{MI}$ in patients presenting with chest pain. However, the improved sensitivity comes along with a decreased specificity, though serial hs-TnI measurements and the detection of early changes could improve the specificity and the overall diagnostic performance. Moreover, apart from their use in the diagnosis and risk stratification of MI and acute coronary syndromes, hs-TnI assays seem to have a key role in risk stratification and short and long-term prognosis in a variety of cardiovascular modalities such as stable coronary disease, heart failure 
and acute pulmonary embolism. In addition, studies have suggested that hs-TnI may be used as a biomarker in the primary prevention of cardiovascular disease leading to the identification of high-risk populations or individuals with silent heart disease.

However, due to assay variability, the deployment of a standardization and harmonization method is required before their wide use in clinical practice. In conclusion, it may be said that these hs-TnI assays will define a high-risk patient population that was not possible with older generation assays and will probably lead to more appropriate therapy and improved outcomes in these patients.

Acknowledgement: We thank Dr Anil Handoo, Director, Department of Laboratory Services, BLK Superspeciality Hospital, Delhi for his encouragement and support in writing this review.

\section{Funding: Nil, Conflict of interest: None. Permission of IRB: Yes}

\section{References}

1. Thygesen K, Alpert JS, White HD; Joint ESC/ ACCF/AHA/WHF Task Force for the Redefinition of Myocardial Infarction. Universal definition of myocardial infarction. Eur Heart J.2007Oct;28(20):2525-38.

2. Morrow DA, Cannon CP, Jesse RL, Newby LK, Ravkilde J, Storrow AB, et al. National Academy of Clinical Biochemistry. National Academy of Clinical Biochemistry Laboratory Medicine Practice Guidelines: Clinical characteristics and utilization of biochemical markers in acute coronary syndromes. Circulation. 2007 Apr 3; 115(13):e356-75. Epub 2007 Mar 23.

3. Gaze DC, Collinson PO. Multiple molecular forms of circulating cardiac troponin: analytical and clinical significance. Ann Clin Biochem. 2008 Jul;45(Pt 4):34955. doi: 10.1258/acb.2007.007229.

4. Apple FS. A new season for cardiac troponin assays: it's time to keep a scorecard. Clin Chem. 2009 Jul; 55 (7):1303-6. doi: 10.1373/clinchem.2009.128363. Epub 2009 May 28.

5. Jesse RL. On the relative value of an assay versus that of a test: a history of troponin for the diagnosis of myocardial infarction. J Am Coll Cardiol. 2010 May 11; 55 (19):2125-8. doi: 10.1016/j.jacc.2010.03.014.
6. Reichlin T, Hochholzer W, Bassetti S, Steuer S, Stelzig C, Hartwiger S, et al. Early diagnosis of myocardial infarction with sensitive cardiac troponin assays. N Engl J Med. 2009 Aug 27;361(9):858-67. doi: 10. 1056/NEJMoa0900428.

7. Thygesen K, Mair J, Giannitsis E, Mueller C, Lindahl B, Blankenberg S, et al; Study Group on Biomarkers in Cardiology of ESC Working Group on Acute Cardiac Care. How to use high-sensitivity cardiac troponins in acute cardiac care. Eur Heart J.2012 Sep;33(18):2252-7. Epub 2012 Jun 21.

8. Wijnker PJ, Li Y, Zhang P, Foster DB, dos Remedios C, Van Eyk JE et al. A novel phosphorylation site, Serine 199, in the C-terminus of cardiac troponin I regulates calcium sensitivity and susceptibility to calpain-induced proteolysis.J Mol Cell Cardiol. 2015 May; 82:93-103. doi: 10.1016/j.yjmcc.2015.03.006. Epub 2015 Mar 11.

9. Lyngbakken MN, Røsjø H, Holmen OL, Nygård S, Dalen H, Hveem K et al. High-Sensitivity Troponin I, and the Risk of Cardiovascular Events (from the Nord-Trøndelag Health Study).Am J Cardiol. 2016 Sep 15; 118(6):816-21. doi: 10.1016/j.amjcard.2016.06.043. Epub 2016 Jun 28.

10. Trambas C, Pickering JW, Than M, Bain C, Nie L, Paul E et al. Impact of High-Sensitivity Troponin I Testing with Sex-Specific Cutoffs on the Diagnosis of Acute Myocardial Infarction.Clin Chem. 2016 Jun; 62 (6):831-8. doi: 10.1373/clinchem.2015.252569. Epub 2016 Apr 26.

11. Bossard M, Thériault S, Aeschbacher S, Schoen T, Kunz S, von Rotz $M$ et al. Factors independently associated with cardiac troponin I levels in young and healthy adults from the general population.Clin Res Cardiol. 2016 Aug 17. (Epub ahead of print).

12. Sandoval Y, Smith SW, Shah AS, Anand A, Chapman AR, Love SA et al. Rapid Rule-Out of Acute Myocardial Injury Using a Single HighSensitivity Cardiac Troponin IMeasurement.Clin Chem. 2016 Nov 3. pii: clinchem.2016.264523. (Epub ahead of print).

13. Love SA, Sandoval Y, Smith SW, Nicholson J, Cao $\mathrm{J}$, Ler R, et al. Incidence of Undetectable, Measurable, and Increased Cardiac Troponin I Concentrations 
Above the 99th Percentile Using a High-Sensitivity vs a Contemporary Assay in Patients Presenting to the Emergency Department. Clin Chem. 2016 Aug;62 (8): 1115-9.doi: 10.1373/clinchem.2016.256305. Epub 2016 Jun 20.

14. Westwood M, van Asselt T, Ramaekers B, Whiting $\mathrm{P}$, Thokala $\mathrm{P}$, Joore $\mathrm{M}$ et al. High-sensitivity troponin assays for the early rule-out or diagnosis of acute myocardial infarction in people with acute chest pain: a systematic review and cost-effectiveness analysis. Health Technol Assess. 2015 Jun;19(44):1-234. doi: 10. 3310 /hta19440.15. Clin Chem. 2016 Mar; 62(3):494504. doi: 10.1373/clinchem. 2015. 249508. Epub 2016 Jan 21.

15. Reichlin T, Cullen L, Parsonage WA, Greenslade J, Twerenbold R, Moehring B, et al. Two-hour algorithm for triage toward rule-out and rule-in of acute myocardial infarction using high-sensitivity cardiac troponin T. Am J Med. 2015 Apr;128(4):369-79.e4. doi: 10.1016/j.amjmed.2014.10.032. Epub 2014 Nov 13.

16. Pickering JW, Young JM, George P, Aldous S, Cullen L, Greenslade JH et al. The utility of presentation and 4-hour high sensitivity troponin I to rule-out acute myocardial infarction in the emergency department.Clin Biochem. 2015 Dec;48(18):1219-24. doi: 10.1016/j.clinbiochem.2015.07.033. Epub 2015 Jul 29.

17. Rubini Gimenez M, Twerenbold R, Jaeger C, Schindler C, Puelacher C, Wildi K, et al. Campodarve I, Rentsch K, Bassetti S, Osswald S, Mueller C. One-hour rule-in and rule-out of acute myocardial infarction using high-sensitivity cardiac troponin I. Am J Med. 2015 Aug; 128(8):861-870.e4. doi: 10.1016/j.amjmed.2015. 01.046. Epub 2015 Mar 31.

18. Cullen L, Greenslade JH, Carlton EW, Than M, Pickering JW, Ho A et al. Sex-specific versus overall cut points for a high sensitivity troponin I assay in predicting 1-year outcomes in emergency patients presenting with chest pain. Heart. 2016 Jan;102(2):1206. doi: 10.1136/heartjnl-2015-308506.

19. Jaeger C, Wildi K, Twerenbold R, Reichlin T, Rubini Gimenez M, Neuhaus JD, et al. One-hour rule-in and rule-out of acute myocardial infarction using highsensitivity cardiac troponin I. Am Heart J. 2016 Jan; 171(1):92-102.e1-5. doi: 10.1016/j.ahj.2015.07.022. Epub 2015 Jul 26.

20. Eggers KM, Aldous S, Greenslade JH, Johnston N, Lindahl B, Parsonage WA et al. Two-hour diagnostic algorithms for early assessment of patients with acute chest pain--Implications of lowering the cardiac troponin I cut-off to the 97. 5th percentile.Clin Chim Acta. 2015 May 20;445:19-24. doi: 10.1016/j.cca. 2015. 03.002. Epub 2015 Mar 11.

21. Hoeller R, Rubini Giménez M, Reichlin $\mathrm{T}$, Twerenbold R, Zellweger C, Moehring B et al. Normal presenting levels of high-sensitivity troponin and myocardial infarction.Heart. 2013 Nov; 99(21): 156772. doi:10.1136/heartjnl-2013-303643. Epub 2013 Apr 19.

22. Schreiber DH, Agbo C, Wu AH. Short-term ( 90 min) diagnostic performance for acute non-ST segment elevation myocardial infarction and 30-day prognostic evaluation of a novel third-generation high sensitivity troponin I assay.Clin Biochem. 2012 Nov;45 (16-17) : 1295-301. doi: 10.1016/j.clinbiochem. 2012. 06.005. Epub 2012 Jun 15.

23. Lyck Hansen M1, Saaby L, Nybo M, Rasmussen LM, Thygesen K, Mickley $\mathrm{H}$ et al. Discordant diagnoses of acute myocardial infarction due to the different use of assays and cut-off points of cardiac troponins.Cardiology. 2012;122(4):225-9. doi: 10.1159/ 000339269. Epub 2012 Aug 10.

24. Iribarren C, Chandra M, Rana JS, Hlatky MA, Fortmann SP, Quertermous T, et al. High-sensitivity cardiac troponin I and incident coronary heart disease among asymptomatic older adults. Heart. 2016 Aug 1;102(15):1177-82. doi: 10.1136/heartjnl-2015-309136. Epub 2016 Mar 30.

25. Thorsteinsdottir I, Aspelund T, Gudmundsson E, Eiriksdottir G, Harris TB, Launer LJ, et al. HighSensitivity Cardiac Troponin I Is a Strong Predictor of Cardiovascular Events and Mortality in the AGESReykjavik Community-Based Cohort of Older Individuals. Clin Chem. 2016 Apr;62(4):623-30. doi: 10.1373/clinchem.2015.250811. Epub 2016 Mar 2.

26. Ferencik M, Liu T, Mayrhofer T, Puchner SB, Lu MT, Maurovich-Horvat P et al. hs-Troponin I Followed by CT Angiography Improves Acute Coronary 
Syndrome Risk Stratification Accuracy and Work-Up in Acute Chest Pain Patients: Results From ROMICAT II Trial. JACC Cardiovasc Imaging. 2015 Nov; 8(11): 1272- 81. doi: 10.1016/j.jcmg.2015.06.016. Epub 2015 Oct 14.

27. Aeschbacher S, Schoen T, Bossard M, van der Lely S, Glättli K, Todd J, et al. Relationship between highsensitivity cardiac troponin I and blood pressure among young and healthy adults. Am J Hypertens. 2015 Jun; 28 (6):789-96. doi: 10.1093/ajh/hpu226. Epub 2014 Nov 25.

28. Yiu KH, Lau KK, Zhao CT, Chan YH, Chen Y, Zhen $Z$ et al. Predictive value of highsensitivity troponin-I for future adverse cardiovascular outcome in stable patients with type 2 diabetes mellitus.Cardiovasc Diabetol. 2014 Mar 25;13:63. doi: 10.1186/1475-2840-13-63.
29. Bargnoux AS, Kuster N, Patrier L, Dupuy AM, Tachon G, Maurice F, et al. Cardiovascular risk stratification in hemodialysis patients in the era of highly sensitive troponins: should we choose between hs-troponin I and hs-troponin T? Clin Chem Lab Med. 2016 Apr; 54 (4):673-82. doi: 10.1515/cclm- 20150071.

30. Mbagaya W, Luvai A, Lopez B. Biological variation of cardiac troponin in stable haemodialysis patients. Ann Clin Biochem. 2015 Sep; 52(Pt 5):562-8. doi: 10.1177/0004563215585877. Epub 2015 Apr 23.

31. McKie PM, Heublein DM, Scott CG, Gantzer ML, Mehta RA, Rodeheffer RJ, et al. Defining highsensitivity cardiac troponin concentrations in the community. Clin Chem. 2013 Jul;59(7):1099-107. doi: 10.1373/clinchem.2012.198614. Epub 2013 Apr 16.

\section{How to cite this article?}

Neogi S.S, Kapoor R.K. hsTropI: an early biomarker of acute coronary syndrome \& MI. Int J Med Res Rev 2017;5(01):80-87. doi:10.17511/ijmrr. 2017.i01.12. 